\title{
Nanometre-scale resolution achieved with Nonradiative Excitation
}

\author{
Lina Riachy, Dalia El Arawi, Rodolphe Jaffiol, and Cyrille Vézy* \\ Lumière Nanomatériaux Nanotechnologies, Institut Charles Delaunay, UMR CNRS 6281, \\ Université de Technologie de Troyes, 12 rue Marie Curie, CS 42060, 10004 Troyes cedex, \\ France. \\ E-mail: cyrille.vezy@utt.fr \\ Phone: +33 (0) 3515911 55. Fax: +33 (0)3 25718456
}

\begin{abstract}
Nonradiative Excitation Fluorescence Microscopy (NEFM) is a promising technique allowing the observation of biological samples beyond the diffraction limit. By coating a substrate with an homogeneous monolayer of quantum dots (QDs), NEFM is achieved through a nonradiative energy transfer from QDs (donors) to dye molecules located in the sample (acceptors). The excitation depth of the sample is then given by the Förster radius, which corresponds to few nanometers above the surface. The powerful axial resolution of NEFM is highlighted by observing the adhesion of Giant Unilamellar Vesicles (GUVs) on strong interaction with coated surfaces. In this paper, we demonstrate that the QD-quenching level is valuable to calculate and map the distance between the membrane and the surface with a high precision along the optical axis. By tuning the electrostatic interactions between the membrane and the substrate, we have been able to measure a height displacement of $\approx 1 \mathrm{~nm}$ of the lipid membrane. The experimental results were discussed according to simulations, which take into account all the common forces appearing between lipid membranes and surfaces.
\end{abstract}




\section{Keywords}

Biophotonics, Förster Resonance Energy Transfer, Membrane, Nanoscopy

Förster Resonance Energy Transfer (FRET) is now a standard technique, widely employed in biophysics and biophtonics. FRET relies on a nonradiative energy transfer from excited donor molecules to acceptor molecules in their ground state. This energy transfer is efficient as long as the distance between the two molecules is less than $10 \mathrm{~nm}$. FRET is usually considered as a nanoscale ruler with a broad area of applications that includes structural biology, ${ }^{1}$ biosensing, ${ }^{2,3}$ binding measurement between molecules ${ }^{4}$ or structure of intermembrane junction. ${ }^{5}$ The signal obtained from FRET is typically examined by spectra, fluorescence pictures or by time-resolved investigations. In this paper, we propose an original imaging method, based on donor quenching analysis, to measure, with a nanoscale accuracy, distances involved in the adhesion of membranes on a surface.

Giant Unilamelar Vesicles (GUVs) are extensively used as biomimetic objects to explore and understand physical basis of cell adhesion ${ }^{6,7}$ and to test new imaging techniques. ${ }^{8,9}$ As membrane composition can be easily modified with several kinds of lipids wearing charges or specific functions, the vesicles spreading will change as their interactions with the substrate change. Different optical tools were proposed to observe and understand the adhesion of lipid membranes or cells. First, Reflection Interference Contrast Microscopy (RICM) ${ }^{10-13}$ and related improvements (dual wavelength, ${ }^{14}$ refined version of RICM theory ${ }^{9}$ )or Fluorescence Interference Contrast (FLIC) microscopy ${ }^{5,15,16}$ allowed quantitative measurements of the membrane-surface distance. Second, microscopy techniques using evanescent wave and its new adaptations, such as normalised Total Internal Reflection Microscopy (nTIRFM) ${ }^{8,17}$ and variable-angle Total Internal Reflection Fluorescence Microscopy (vaTIRFM), ${ }^{18}$ are also well suited to observe and quantify the adhesion of living cells or GUVs near a wall. However, all these techniques do not allow the obervation of membrane nanometric displacement, related to tiny modulations of the membrane/substrate interactions. This is mainly due to the axial resolution of these usual techniques, which is, on soft material at room temperature, at best 
$\approx 5 \mathrm{~nm}$ for RCIM and 5-10 $\mathrm{nm}$ for fluorescent technique. To measure membrane/substrate interdistance with a nanometric accuracy, we have recently proposed an appealing alternative to interferometry or Total Internal Reflection, based on FRET. This nanoimaging technique is called Nonradiative Excitation Fluorescence Microscopy (NEFM). ${ }^{19}$ NEFM is based on the activation of glass coverslip with quantum dots (QDs). This was firstly achieved through the deposition of a thin layer of PMMA highly doped with QDs. ${ }^{19}$ However, PMMA dewetting occurred after few hours or less, and therefore long time observations were not possible anymore. Moreover, the QDs density in PMMA cannot be controled properly. In order to overcome these limitations, glass coverslips were activated by adding a monolayer of CdSe/ZnS quantum dots (QDs) with a silanisation process. ${ }^{20}$ To highlight the powerful axial resolution of NEFM and the benefits of this new substrate preparation, we observed GUV spreading on glass substrate coated with QDs, when adhesion is mediated by electrostatic interactions. To this end the QDs were surrounded by a positively charged polymer, Poly-L-Lysine (PLL), and membranes were labeled with a negatively charged dye (DiD). The excitation depth in NEFM is typically $\approx 10 \mathrm{~nm}$ at the vicinity of the surface. This corresponds to two times the Förster radius. According to the FRET theory and by recording both QDs-quenching and fluorescence induced by FRET, a distance map between the surface and the lipid membrane can be reconstructed according to a straightforward image analysis compared to others methods. For example, in RICM (even in dual-wave length RICM) ${ }^{14,21}$ all refractive index should be known to get quantitative informations. Moreover, the data processing is very complex. FLIC or intermembrane FRET methods ${ }^{5,15,16,22}$ also involved a complex data processing and the conversion from FRET efficiency to a distance is difficult. In contrast, with our method, the absolute distance in each pixel of the picture can be obtained easily.

In this paper, we exploit NEFM to study the adhesion of GUVs mainly driven by van der Waals attraction and electrostatic attraction or repulsion. By tuning the strength of electrostatic attraction on the membrane adhesion, we achieved a $1 \mathrm{~nm}$ axial resolution with NEFM. 
This constitutes a major breakthrough in nanoimaging. Until now, only the recent publication about cryogenic single molecule localization microscopy offers a sub-nanometer optical resolution. ${ }^{23}$ At the opposite, more polular techniques such as STED, ${ }^{24}$ single molecule localization combined with supercritical angle ${ }^{25-27}$ or structured illumination ${ }^{28}$ or MIET, ${ }^{29}$ only reach a resolution $\approx 10 \mathrm{~nm}$ at best, at room temperature. NEFM enables to overcome the $10 \mathrm{~nm}$ resolution barrier mainly because nonradiative energy transfer occurs between 1 and $10 \mathrm{~nm}$.

\section{Results and discussion}

\section{Surface characterization}

We characterized our QDs layer on glass coverslips by SEM. The Fig.1 presents a SEM picture of QDs bound on silicon wafer (SEM images on glass coverslips are similar in term of homogeneity, but less contrasted due to glass substrate). This picture indicates that only a monolayer of QDs was achieved at a large scale. Thus our new method of preparation allowed us to create a homogenous surface coated with QDs. According to Fig.1, the surface coverage is about $25 \%$ with a density of QDs about 5000 QDs $/ \mu \mathrm{m}^{2}$. This high surface density gives rise to a homogenous photoluminescent signal within a diffraction limited spot (no blinking was observed). This point is crucial for quantitative analysis in NEFM (the measured fluorescence fluctuations should be induced only by distance changes between donor and acceptor and not by QDs blinking). To exploit FRET theory, the quantum yield $\left(\phi_{D}\right)$ of our surface has to be estimated. The quantum yield of QDs change drastically as a function of solvents or environment. $\phi_{D}$ is given by $\mathrm{Eq} 1$ where $k_{r}$ is the radiative decay rate and $k_{n r}$ is the non-radiative decay rate of QDs excited state.

$$
\phi_{D}=\frac{k_{r}}{k_{r}+k_{n r}}
$$


Table 1: Values of $k_{r}, \tau$ and $k_{n r}$ to calculate the quantum yield $\phi_{D}$ of QDs in toluene solution or deposited on a surface and surrounded with PLL

\begin{tabular}{lllll}
\hline & $k_{r}\left(\mathrm{~ns}^{-1}\right)$ & $\tau(\mathrm{ns})$ & $k_{n r}\left(\mathrm{~ns}^{-1}\right)$ & $\phi_{D}$ \\
\hline QDs in toluene solution & 0.0478 & 16.7 & 0.012 & 0.8 \\
QDs in water & 0.0338 & - & - & - \\
QDs monolayer surrounded with PLL at glass/water interface & 0.0399 & 8 & 0.0851 & 0.32 \\
\hline
\end{tabular}

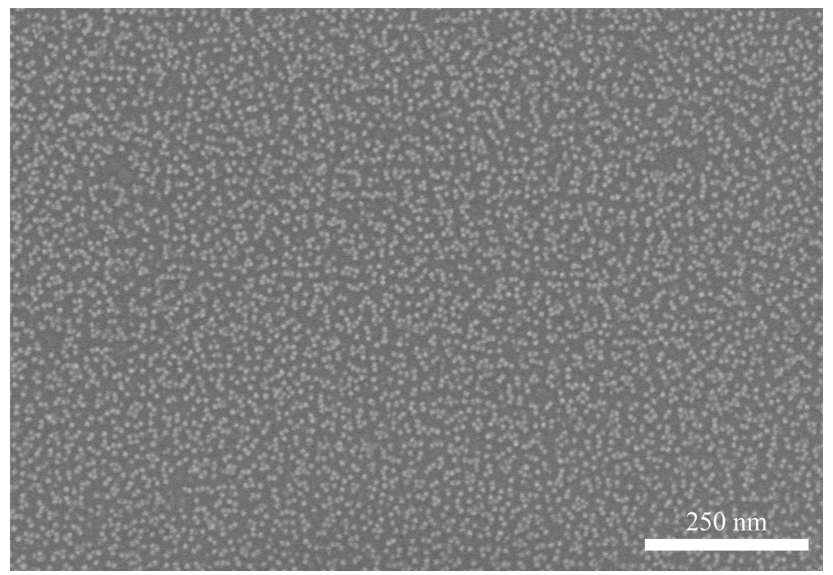

Figure 1: Typical SEM picture of surface coated with CdSe/ZnS QDs.

From the molar extinction spectra and emission spectra, ${ }^{30}$ we estimated $\mathrm{k}_{r}$ for QDs in toluene by using the Strickler and Berg formula. ${ }^{31-33}$ From this value, we have calculated $\mathrm{k}_{r}$ in water. ${ }^{34}$ Then $\mathrm{k}_{r}$ is calculated for QDs monolayer at glass/water interface. ${ }^{35}$ These results are shown in Table 1 . Then $k_{n r}$ is calculated by subtracting $k_{r}$ from the value of the inverse of the measured fluorescence lifetime $(\tau)$ of QDs (Table 1). An example of lifetime measurements is presented in Fig. $\mathrm{S}_{1}$. We have determined the mean lifetime as proposed by A. I. Chizhik, I. Gregor, and J. Enderlein. ${ }^{36} \phi_{D}$ was estimated according to equation 1. $\phi_{D}$ $\approx 0.32$ for our surface covered with QDs and with Poly-L-Lysin while $\phi_{D} \approx 0.8$ when QDs are in a toluene solution (as indicated by Sigma-Aldrich). 


\section{From QDs quenching and NEFM images to distance images}

\section{Calculation of $\mathbf{R}_{0}$, the Förster radius}

The efficiency $E_{t}$ of FRET process can be characterized according to:

$$
E_{t}=\frac{1}{1+\left(\frac{R}{R_{0}}\right)^{6}}=1-\frac{S_{D+A}}{S_{D}}
$$

where $\mathrm{R}$ is the distance between donor and acceptor, $\mathrm{R}_{0}$ is the Förster radius, $S_{D}$ is the signal emitted by donors without acceptors and $S_{D+A}$, the signal emitted by donors when acceptors are added. To validate the relevance of equation 2 in our experiment, we have performed FRET experiments by controlling the distance between the QDs layer and a DiD layer. The FRET efficiency was then calculated and plotted as a function of the distance $\mathrm{R}$ $\left(\right.$ Fig $\mathrm{S}_{2}$ ). These data can be fitted according to three different models : one acceptor coupled with one donor as depicted in equation 2, $\mathrm{n}$ acceptors coupled with one donor and a plane of acceptors coupled with one donor(see Supplementary information). From the obtained results, we have choosen the model when one acceptor is coupled with one donor. Then $\mathrm{R}_{0}^{6}$ (in $\mathrm{cm}^{6}$ ) need to be known and is calculated as following:

$$
R_{0}^{6}=\frac{9(\ln 10) \phi_{D} \kappa^{2}}{128 \pi^{5} n^{4} N_{a}} J(\lambda)
$$

with $\phi_{D}$ the quantum yield, $N_{a}$ the Avogadro number, $\kappa^{2}$ the dipole orientation factor, $\mathrm{n}$ the refractive index of the medium (1.338 for glucose solution). The spectral overlap integral $\mathrm{J}(\lambda)$ between donor and acceptor was numerically calculated according molar extinction and emission spectra (note that the maximum of DiD molar extinction coefficient is 210000 $\mathrm{M}^{-1} \mathrm{~cm}^{-1}$ at $\left.457 \mathrm{~nm}^{37}\right) . \kappa^{2}$ is calculated from R. E. Dale, J. Eisinger, W. E. Blumberg. ${ }^{38}$ DiD dipole orientation is perpendicular to the optical axis ${ }^{39}$ and QDs dipole orientation is randomly distributed. Therefore, the angle between the DiD dipole and the axis defined by donors and acceptors is equal to $\pi / 2$. Then $\kappa^{2}=1 / 3$. So the value of $R_{0}$ for our QDs surface 
surrounded by Poly-L-Lysine at glass/water interface in interaction with a DiD layer is equal to $5.82 \mathrm{~nm}$.

\section{Quenching and fluorescence images}

As vesicles contain sucrose $(200 \mathrm{mM})$, and the surrounding medium is glucose at $250 \mathrm{mM}$ vesicles gently settled on the Poly-L-Lysin/QDs surface due to gravitational force. When GUVs encounter the surface, lysis of all GUVs was observed in pure water due to the strong electrostatic attraction between the surface and GUVs. ${ }^{8}$ Therefore, only supported membranes are observed on the surface. As previously explained, the QDs layer is surrounded with Poly-L-Lysin in order to obtain positively charged surfaces. This charge depends on the salt concentration of the medium (Fig. $\mathrm{S}_{3}$ ). At the opposite, the vesicles are labeled with DiD which is negatively charged. DiD charge does not change according to the salt concentration (as the pKa of the benzene sulfonic acid of DiD is at 2.54, and the $\mathrm{pH}$ working range in our experiments is higher than 6 , the benzene sulfonic acid always exhibit a negative charge). Fig.2 shows a supported lipid bilayer on the surface observed i NEFM at $457 \mathrm{~nm}$ and TIRF at $633 \mathrm{~nm}$. First, the TIRF illumination indicates the GUV lysis (no more vesicles were observed with defocusing imaging) (Fig. 2.a). Next, All experiments were performed with a laser irradiance of $50 \mathrm{~mW} / \mathrm{cm}^{2}$ at $457 \mathrm{~nm}$ and an acquisition time of $300 \mathrm{~ms}$. At this irradiance, no direct excitation at $457 \mathrm{~nm}$ of the acceptor (DiD) is observed. As a consequence, the fluorescence emission of $\mathrm{DiD}$ (Fig.2b) is only due to FRET process. ${ }^{20}$ In the spectral window of the QDs emission (Fig.2.c), quenching appears where DiD emission (Fig.2.b) is expected. Spectra recorded on QDs with and without supported lipid layer also confirms that FRET occurs (Fig.S $\left.{ }_{4}\right)$. The fluorescence emitted by DiD through FRET is very homogenous without any fluctuation in time. Undulations of the membrane (related to Helfrich force) are suppressed due to the strong electrostatic attraction. Only photobleaching was observed with a slow decay rate $(\approx 60 \mathrm{~s})$. The Fig.3 presents a zoomed representation of our system. Due to the presence of octadecylamine ligands on the QDs, the PLL is deposited between 

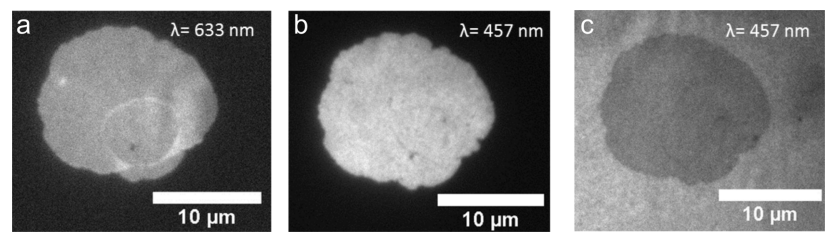

Figure 2: Lysis of vesicles on a QDs layer surrounded by Poly-L-Lysine. a: TIRF image at $633 \mathrm{~nm}$. b: fluorescence emission of DiD excited at $457 \mathrm{~nm}$ (spectral detection window : 670-694 nm). c: photoluminescence of QDs excited at at $457 \mathrm{~nm}$ (spectral detection window : 490-610 nm).

the QDs, on the glass substrate, helped by electrostatic attraction between the amine groups of PLL and silanol groups on glass. Because it was diluted in a low concentration $\mathrm{NaOH}$ aqueous solution (i.e. poor solvent), in these conditions, PLL opted for a mushroom-like configuration as depicted in Fig.3. PLL hydrodynamic radius is about $5 \mathrm{~nm}$ in solution, so the thickness of PLL is similar or smaller than the QDs diameter. ${ }^{40}$ Moreover the fluorescence observed on the lipid bilayer is laterally homogenous (Fig.2.b). This means that the lipid bilayer seems to be flat above the QD layer.

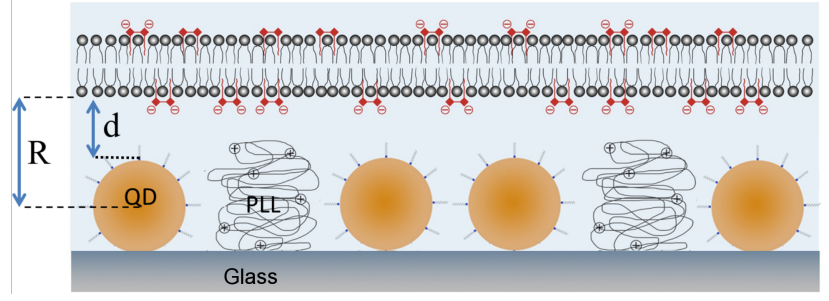

Figure 3: Schematic view of the studied system. $\mathrm{R}$ is the distance between the center of the QDs layer to the lipid membrane. $d$ is the distance from the top of the QDs layer to the lipid membrane. $\mathrm{d}_{0}$ is the corresponding equilibrium distance.

From the equation (2) and with image recorded through the detection spectral window of QDs, we have calculated the efficiency of FRET transfer, $\mathrm{E}_{t}$, for each pixels. We used the NEFM image to determine on which pixel the transfer efficiency must be calculated because its contrast is very good. As a result, the fluorescence image is converted into a map of the normalized distance, denoted $\mathrm{R} / \mathrm{R}_{0}$, between the surface and the membrane (Fig.4). From this map, we have also calculated the histogram of $R / R_{0}$. The mean value $\left(<R / R_{0}>\right)$ and 


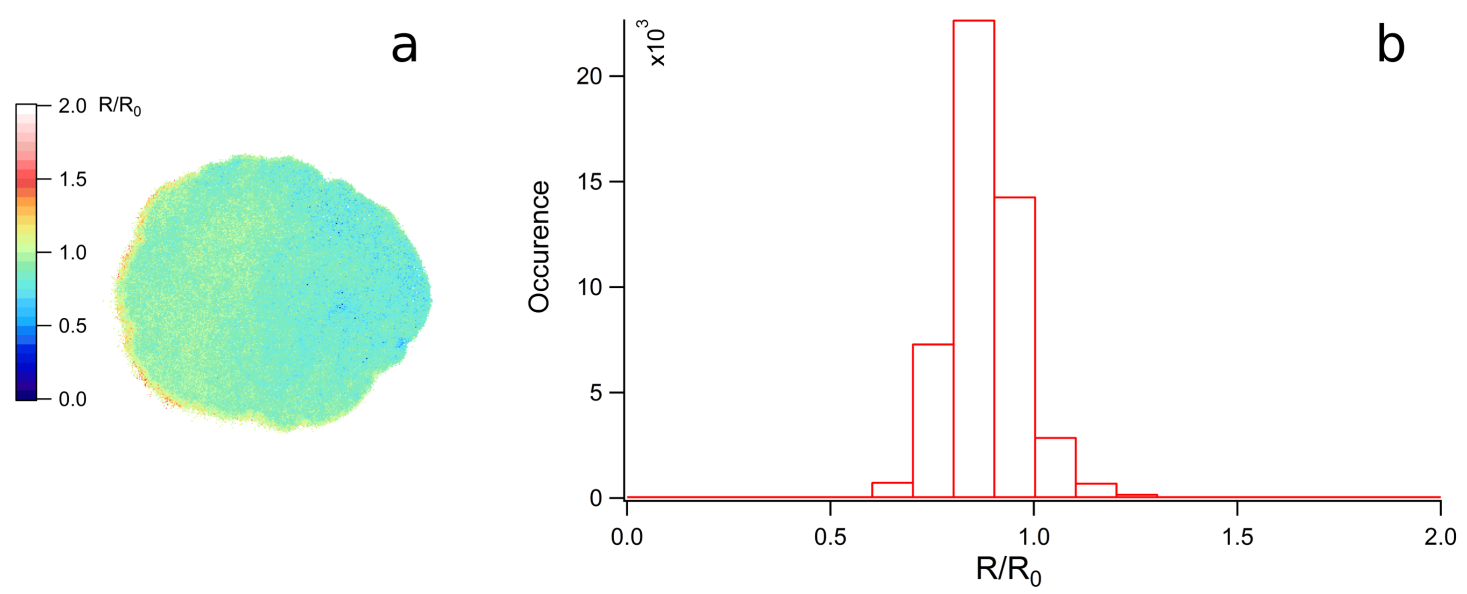

Figure 4: a. $\mathrm{R} / \mathrm{R}_{0}$ distance calculated from the data shown in Fig.3. b: Corresponding distance histogram.

the standard deviation $(\sigma)$ were obtained according a Gaussian model. $\sigma$ takes into account the height of the membrane fluctuation above the surface and also the heterogeneity of the functionalized surface. As shown in Fig.4, in pure water at $\mathrm{pH}=6,<\mathrm{R} / \mathrm{R}_{0}>=0.83$ and $\sigma$ $=0.003$. If we used the value previously calculated for $\mathrm{R}_{0}=5.82 \mathrm{~nm}$, the mean distance $<\mathrm{R}>$ $=4.83 \mathrm{~nm}+/-0.02 \mathrm{~nm}$. R corresponds to the distance from the center of the QDs to the lipid membrane. Therefore, the water gap thickness from the top of QDs to the membrane is $\approx 1 \mathrm{~nm}$ (the QDs radius is $4 \mathrm{~nm}$ as evaluated by SEM).

\section{Influence of the addition of salt on the equilibrium distance $d_{0}$}

In order to modulate the strength of the electrostatic attraction and measure how this modulation affects the equilibrium distance between the surface and the lipid bilayer, sodium hydroxide was added in the glucose solution. The salt concentration varied from 0 to 233 $\mu \mathrm{M}$. Below a concentration of $70 \mu \mathrm{M}$, lysis of all vesicles is observed (Fig.5). On Fig.5 $\mathrm{A}_{2}$, $\mathrm{A}_{4}$, we observed the formation of a stack of lipid bilayers due to the presence of small vesicles inside bigger one. This can be easily checked on TIRF pictures recorded at $633 \mathrm{~nm}$ where brighter zones appear in the center. The fluorescence of the DiD is twice higher in the center. This is the signature of the presence of two lipid bilayers stacked in the center of 

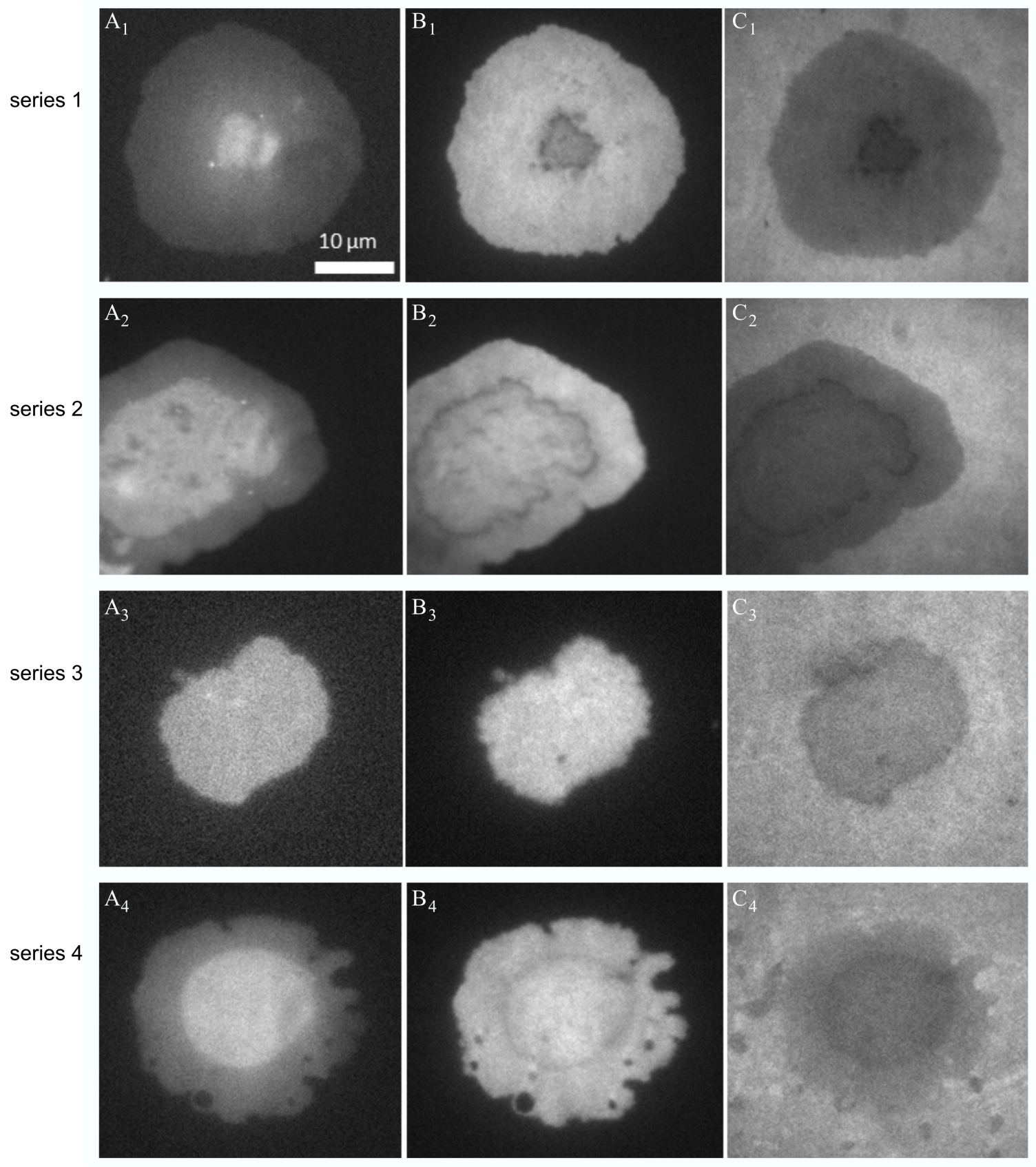

Figure 5: Images of supported lipid membrane for different concentration of $\mathrm{NaOH}$ (series 1 : $23 \mu \mathrm{M}$, series $2: 35 \mu \mathrm{M}$, series $3: 47 \mu \mathrm{M}$, series $4: 58 \mu \mathrm{M})$ observed in TIRF illumination at $633 \mathrm{~nm}\left(\mathrm{~A}_{i}\right)$. Lipid bilayers are also excited at $457 \mathrm{~nm}$ and the emission of $\mathrm{DiD}\left(\mathrm{B}_{i}\right)$ and the quenching of QDs layer $\left(\mathrm{C}_{i}\right)$ were collected with relevant band-pass filters.

the structure while only a single lipid bilayer is obtained at the periphery. At the junction of the bilayer and the quadrilayer, the QDs quenching is stronger which could be due to a decrease of the distance (Fig. $5 \mathrm{C}_{2}, \mathrm{C}_{4}$ ). At the same time, the DiD emission in NEFM 
images is lower due to a depletion of $\mathrm{DiD}$ during the formation of this structure (Fig. $5 \mathrm{~B}_{2}$, $\mathrm{B}_{4}$ ). In the center, the $\mathrm{DiD}$ fluorescence recorded in NEFM seems to be not affected by the presence of the second bilayer. This clearly demonstrates that the second bilayer is out of the range of FRET process and its distance is greater than $10 \mathrm{~nm}$ from the center of the QDs layer. Indeed, the second bilayer is repelled by the first one, due to electrostatic repulsion as both bilayers were negatively charged. On the series 3 , only a single bilayer is observed. On the series 1 , the lipid organization in the center should be different than a simple stack of two lipid bilayer as the signal in NEFM decreases (Fig.5 $\mathrm{B}_{1}$ ). This should be due to a depletion of DiD with a decrease of the distance. To avoid such artefacts related to multistack, we have analyzed zones where only one lipid bilayer is obtained (usually at the periphery of the structures). As previously, we have calculated from the quenching of QDs and according to the Equation 2, the ratio $R / R_{0}$ for several lysed vesicles (at least 10 vesicles for each sodium hydroxide concentration). Histograms of these distances are plotted in Fig.6. All histograms were fitted with a Gaussian model to get the mean value $\left(<R / R_{0}>\right)$
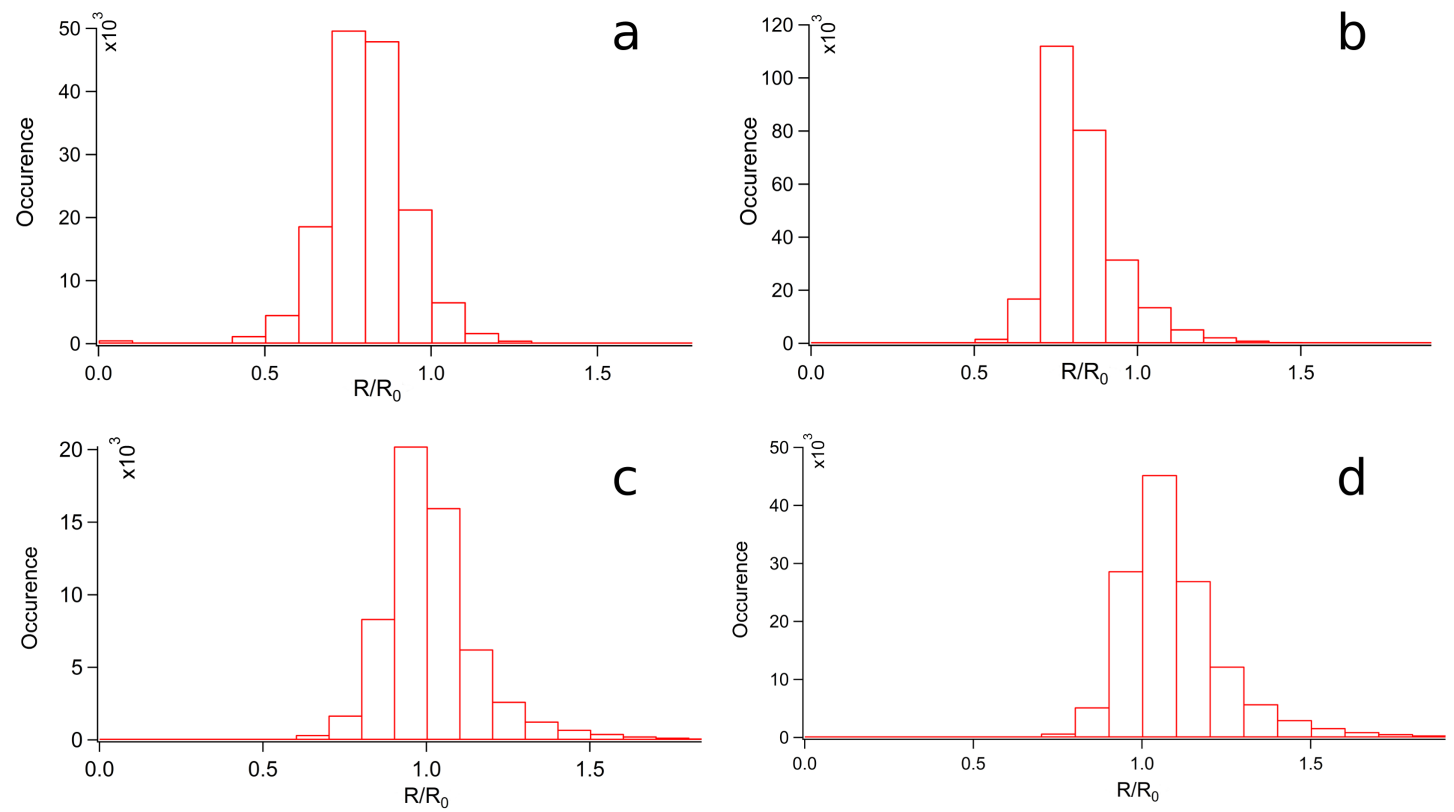

Figure 6: Histograms of $\mathrm{R} / \mathrm{R}_{0}$ distance obtained on at least ten vesicles at different $\mathrm{NaOH}$ concentrations (a: $23 \mu \mathrm{M}, \mathrm{b}: 35 \mu \mathrm{M}, \mathrm{c}: 47 \mu \mathrm{M}$ and $\mathrm{d}: 58 \mu \mathrm{M}$ ).

and the standard deviation $(\sigma)$. We observed clearly a shift to higher value when the $\mathrm{NaOH}$ 
Table 2: Evolution of the spreading factor $(\mathrm{S})$ measured with TIRFM and NEFM pictures as a function of the concentration of $\mathrm{NaOH}$

\begin{tabular}{lll}
\hline$[\mathrm{NaOH}](\mu \mathrm{mol} / \mathrm{L})$ & $\mathrm{S}(\mathrm{TIRF})$ & $\mathrm{S}(\mathrm{NEFM})$ \\
\hline 87 & 0.66 & 0.23 \\
116 & 0.50 & 0.13 \\
\hline
\end{tabular}

concentration is increased. The water gap thickness increases wih the salt concentration. Since $\mathrm{R}_{0}=5.82 \mathrm{~nm}$, we have plotted the evolution of $\left\langle\mathrm{d}_{0}>\right.$ as a function of the sodium hydroxide concentration (Fig.7). As the concentration increase, the interdistance $\mathrm{d}_{0}$ from

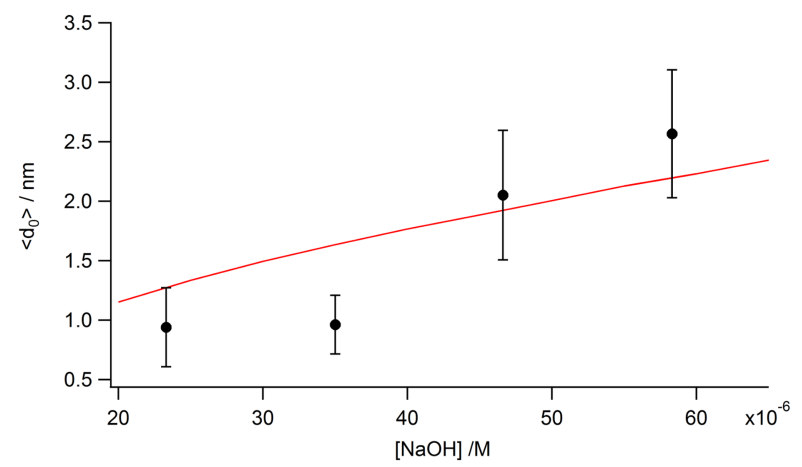

Figure 7: Variation of the average equilibrium inter distance $\mathrm{d}_{0}$, as function of salt concentration. In red, the equilibrium distance calculated from the simulations (see supporting informations).

the top of the QDs to the lipid membrane increases from $0.94 \mathrm{~nm}$ to $2.57 \mathrm{~nm}$.

For salt concentration between $87 \mu \mathrm{M}$ and $120 \mu \mathrm{M}$, we observed vesicles in adhesion without any lysis (Fig. $\left.S_{5}\right)$. As previously proposed, ${ }^{17}$ we have estimated the apparent diameter (D) of GUVs in adhesion in this case. From TIRF and NEFM images we have measured a contact area, denoted $\mathrm{C}$, and then calculated a spreading factor, denoted $\mathrm{S}$, for both excitation cases $\left(\mathrm{S}=\mathrm{C} /\left(\pi(\mathrm{D} / 2)^{2}\right)\right)$. As we can see in Table $2, \mathrm{~S}$ decreases as the salt concentration increases. Therefore, the mean equilibrium distance $\mathrm{d}_{0}$ continues to increase as a function of the salt concentration. For salt concentrations higher than $120 \mu \mathrm{M}$, all vesicles are repelled far away from the surface and no fluorescence can be observed in NEFM imaging (S is lower than 0.1 as indicated by TIRF imaging). These results clearly indicate that the equilibrium distance $\mathrm{d}_{0}$ between the membrane and the QDs continues to increase as a function of the 
salt concentration.

To uncover which forces induce such behavior, we have performed simulations. In the case of GUVs lysis, we have to consider 3 forces: van der Waals attraction, electrostatic attraction and steric repulsion mediated by PLL and QDs ligands. These forces are detailed in the Supporting Information. For the electrostatic force, the surface potential and the potential of the lipid bilayer have been estimated. To characterize the potential of the functionalized glass coverslips, we measured the $\zeta$-potential with a Zetasizer (Malvern, Nano-ZS) of micronic silica particles which exhibit the same surface functionalization with PLL. Note that the QDs are neutral and do not contribute to the effective charge of the surface. The $\zeta$-potential was measured as a function of the salt concentration. We obtained that the $\zeta$-potential is positive when the concentration of $\mathrm{NaOH}$ is lower than a threshold value and becomes negative for higher values of the salt concentration (Fig. $\left.\mathrm{S}_{3}\right)$. More we add salt and more the positive charges on the PLL are screened and therefore the surface potential decrease. This decrease was modeled by using the Graham equation (see supporting information) and therefore the surface potential was calculated as a function of the salt concentration (Table $\mathrm{S}_{1}$ ). We have also measured the $\zeta$-potential of the lipid bilayer on Small Unilamelar Vesicles (SUVs). Their potential appears to be always negative and its value is around $-25 \mathrm{mV}$. From all this data, one can calculate, for a given salt concentration the evolution of the potential energy of membrane/substrate interactions resulting from these three forces as a function of the distance between the surface and the lipid bilayer (Fig. $\mathrm{S}_{7}$ ). The equilibrium distance $\mathrm{d}_{0}$ can be easily deduced, as the minimum of the potential energy. This was done for various salt concentrations and $\mathrm{d}_{0}$ was plotted together with our experimental data (Fig.7). We obtained a relative good agreement between this model and our experimental results. In both cases an increase of $\approx 1 \mathrm{~nm}$ of the equilibrium distance is achieved. The small jump observed in the experimental data points could be due to an abrupt increase in the gyration radius of repeller molecules when the salt concentration increases (only a gradual incease is taking into account in the simulations). 
In the case where no lysis of vesicles was observed, two more forces must be considered. The first one is the gravitational attraction as vesicles have sucrose inside and are suspended in glucose solution. The difference of density between sucrose and glucose induce the sedimentation of vesicles. The expression of this attractive potential is given in the supporting information. The second force is the Helfrich repulsion and its expression is also given in supporting information. Lipid membranes fluctuated freely in bulk, but when GUVs are closed to a surface, the thermal fluctuation spectrum changed and it yielded to a repulsive force. We have showed, as previously observed ${ }^{17}$ that the equilibrium distance is about few hundreds nanometers (Fig. $\mathrm{S}_{8}$ ), which is in good agreement with our experimental results.

\section{Materials and Methods}

\section{Materials}

CdSe/ZnS quantum dots (748099), (3-mercaptopropyl)trimethoxysilane (175617), poly-Llysine solution (P4707) and DOPC (1,2-Dioleoyl-sn-glycero-3-phosphocholine, P6354) were purchased from Sigma-Aldrich. DiD (DilC18(5)-DS (1,1'-Dioctadecyl-3,3, 3, 3'-Tetramethylindodicarbocyanine-5,5'-Disulfonic Acid, D12730) was purchased from ThermoFisher Scientific. Thickness-corrected glass coverslip $(170 \mu \mathrm{m}+/-10 \mu \mathrm{m})$ were purchased from Assistant, Sondheim, Germany.

\section{Giant Unilamellar Vesicles (GUVs) preparation}

GUVs were prepared using the standard electroformation method. We mixed DOPC (2 $\mathrm{mg} / \mathrm{mL}$ in methanol/chloroform (9:1)) with DiD (18 $\mu \mathrm{M}$ in methanol/chloroform). The lipid membrane is negatively charged due to the presence of two benzene sulfonic acid groups on DiD. $50 \mu \mathrm{L}$ of the mixture is deposited on two ITO (Indium Tin Oxide) conducting plates. After evaporation of the solvent in a vacuum chamber, the plates were fixed face to face in a teflon holders in order to make a hermetic chamber. A $200 \mathrm{mM}$ sucrose solution at $\mathrm{pH} 6$ 
was injected to hydrate lipids. Then an alternative electric field was applied between the two ITO plates at $10 \mathrm{~Hz}$. The electric field intensity was increased from $0.2 \mathrm{~V}$ to $0.8 \mathrm{~V}$ and maintained overnight. Later, the electric field was decreased to $0.2 \mathrm{~V}$ at $4 \mathrm{~Hz}$ for 20 minutes. Then, GUVs were gently removed from the ITO plates with a thick needle syringe (18G) to avoid lysis.

\section{Surface preparation}

Prior to any chemical treatment, glass coverslips were cleaned by immersion in a Piranha Solution $\left(50 \% \mathrm{H}_{2} \mathrm{SO}_{4}, 50 \% \mathrm{H}_{2} \mathrm{O}_{2}\right)$ for 30 minutes and rinsed extensively with pure water. S1813 photoresist was added between two coverslips to cover one side of coverslips, leaving the other side free for the silanization. Then, this new structure was incubated in the (3-mercaptopropyl)trimethoxysilane diluted in anhydrous toluene at $0.01 \%$ for 12 hours. Afterwards, the substrate was immersed in a solution of quantum dots (QDs) in toluene $(0.08 \mathrm{mg} / \mathrm{L})$ for 24 hours. At last, the substrate is rinsed with toluene and next with acetone

in order to remove the S1813 photoresist. At the end, one can obtain 2 functionalized coverslips with QDs on one side. All reactions were performed in a glove box under argon gas. Then the coverslips were incubated for $60 \mathrm{~min}$ in a poly-L-lysine solution $(0.01 \% \mathrm{w} / \mathrm{v})$.

\section{Microscopy}

Our set-up was extensively described in a previous paper. ${ }^{20}$ Briefly, two laser beams were implemented (one at $457 \mathrm{~nm}$ for QDs excitation, and one at $633 \mathrm{~nm}$ for DiD excitation). A 100 x Zeiss objective (plan-achromat, $\mathrm{NA}=1.46$ ) was used to create a widefield illumination. A rotatable mirror was mount to switch between Total Internal Reflection Fluorescence (TIRF) and epi-illumination. Band-pass filters (Semrock FF01-600/14 and Semrock FF01$682 / 14$ ) were added to observe the QDs emission (the spectrum window is $593 \mathrm{~nm}-607 \mathrm{~nm}$ ) or the DiD emission (spectrum window is $670-694 \mathrm{~nm}$ ). The camera used in all experiments was a CoolSNAP HQ2 (Photometrics Scientific ). 


\section{Conclusion}

We have studied adhesion of vesicles using non-radiative excitation fluorescence microscopy (NEFM). This original approach is based on a FRET process between a surface coated with a monolayer of QDs and fluorescence probes present in the sample. From the quenching of the QDs, the interdistance between the surface and the membrane was assessed for diffeérent adhesion strength. The high sensitivity of FRET allows us to determine variation of the equilibrium distance with $\approx 1 \mathrm{~nm}$ precision along the optical axis. Compared to other super-resolution techniques like STORM, ${ }^{25-27} \mathrm{PALM}^{28} \mathrm{STED}^{24}$ or MIET, ${ }^{29,41}$ NEFM does not need long time acquisition, complex data processing or high laser power. Samples can therefore be illuminated for a long time with a negligible photobleaching. Moreover this technique can be easily implemented on any kind of inverted microscopes. Then, dynamical aspects of cell adhesion and migration can be studied as the acquisition time is typically few hundreds ms. NEFM is also relevant to study th interaction and diffusion of supported lipid bilayers on nanoroughness surfaces. ${ }^{42}$ Finally, this method is also well suited to study biological samples as it was previously demonstrated. ${ }^{19}$ We believe that our technic can be used to probe the dynamic of focal adhesions and also localize precisely cell integrins involved in the focal adhesion plaques.

\section{Acknowledgement}

This work was supported in part by the Grand Troyes, the Conseil départemental de l'Aube, the Fond Européen de Développement Régional (CELLnanoFLUO project).

\section{Supporting Information Available}

The following files are available free of charge. We provide further details on lifetime measurements of QDs, the justification on the FRET law used, the determination of the surface 
potential, the FRET evidence, the adhesion of GUVs, the expression of the different forces involved in the surface/membrane interations and the total interaction potential in case of strong interaction or in case of electrostatic repulsion.

\section{Author Contributions}

L. R. designed the NEFM setup, performed experiments and data analysis. D. E. A performed measurements on the determination of surface potential. R. J. designed the NEFM setup, supervised experiments, performed lifetime measurements and developed the theory. C. V. performed and supervised experiments and developed the data processing routine.

\section{References}

(1) Kalinin, S.; Peulen, T.; Sindbert, S.; Rothwell, P. J.; Berger, S.; Restle, T.; Goody, R. S.; Gohlke, H.; Seidel, C. A. A toolkit and benchmark study for FRET-restrained highprecision structural modeling. Nat. Methods 2012, 9, 1218-1225.

(2) Miyawaki, A.; Llopis, J.; Heim, R.; McCaffery, J. M.; Adams, J. A.; Ikura, M.; Tsien, R. Y. Fluorescent indicators for $\mathrm{Ca} 2+$ based on green fluorescent proteins and calmodulin. Nature 1997, 388, 882-887.

(3) Hildebrandt, N.; Spillmann, C. M.; Algar, W. R.; Pons, T.; Stewart, M. H.; Oh, E.; Susumu, K.; Díaz, S. A.; Delehanty, J. B.; Medintz, I. L. Energy Transfer with Semiconductor Quantum Dot Bioconjugates: A Versatile Platform for Biosensing, Energy Harvesting, and Other Developing Applications. Chemical Reviews 2017, 117, 536-711.

(4) Hodgson, L.; Spiering, D.; Sabouri-Ghomi, M.; Dagliyan, O.; DerMardirossian, C.; Danuser, G.; Hahn, K. M. FRET binding antenna reports spatiotemporal dynamics of GDI-Cdc42 GTPase interactions. Nature Chemical Biology 2016, 12, 802-809. 
(5) Kaizuka, Y.; Groves, J. T. Structure and Dynamics of Supported Intermembrane Junctions. Biophys. J. 2004, 86, 905-912.

(6) Limozin, L.; Sengupta, K. Modulation of vesicle adhesion and spreading kinetics by hyaluronan cushions. Biophys. J. 2007, 93, 3300-3313.

(7) Vézy, C.; Massiera, G.; Viallat, A. Adhesion induced non-planar and asynchronous flow of a giant vesicle membrane in an external shear flow. Soft Matter 2007, 3, 844-851.

(8) Dos Santos, M. C.; Déturche, R.; Vézy, C.; Jaffiol, R. Axial nanoscale localization by normalized total internal reflection fluorescence microscopy. Opt. Lett. 2014, 39, 869-872.

(9) Huang, Z.-H.; Massiera, G.; Limozin, L.; Boullanger, P.; Valignat, M.-P.; Viallat, A. Sensitive detection of ultra-weak adhesion states of vesicles by interferometric microscopy. Soft Matter 2010, 6, 1948-1957.

(10) Zilker, A.,; Engelhardt, H.,; Sackmann, E., Dynamic reflection interference contrast (RIC-) microscopy : a new method to study surface excitations of cells and to measure membrane bending elastic moduli. J. Phys. France 1987, 48, 2139-2151.

(11) Rädler, J.; Sackmann, E. Imaging optical thicknesses and separationdistances of phospholipid vesicles at solid surfaces. J. Phys. II 1993, 3, 727-748.

(12) Bereiter-Hahn, J.; Fox, C. H.; Thorell, B. Quantitative reflection contrast microscopy of living cells. J. Cell Biol. 1979, 82, 767-779.

(13) Geiger, B.; Avnur, Z.; Kreis, T. E.; Schlessinger, J. In The Cytoskeleton; Shay, J. W., Ed.; Springer US: Boston, MA, 1984; pp 195-234.

(14) Schilling, J.; Sengupta, K.; Goennenwein, S.; Bausch, A.; Sackmann, E. Absolute interfacial distance measurements by dual-wavelength reflection interference contrast microscopy. Phys. Rev. E 2004, 69, 1-9. 
(15) Braun, D.; Fromherz, P. Fluorescence interference-contrast microscopy of cell adhesion on oxidized silicon. Appl. Phys. A Mater. Sci. Process. 1997, 65, 341-348.

(16) Kaizuka, Y.; Groves, J. T. Hydrodynamic damping of membrane thermal fluctuations near surfaces imaged by fluorescence interference microscopy. Phys. Rev. Lett. 2006, $96,1-4$

(17) Cardoso Dos Santos, M.; Vézy, C.; Jaffiol, R. Nanoscale characterization of vesicle adhesion by normalized total internal reflection fluorescence microscopy. Biochim. Biophys. Acta - Biomembr. 2016, 1858, 1244-1253.

(18) Cardoso Dos Santos, M.; Déturche, R.; Vézy, C.; Jaffiol, R. Topography of Cells Revealed by Variable-Angle Total Internal Reflection Fluorescence Microscopy. Biophys. J. 2016, 111, 1316-1327.

(19) Winckler, P.; Jaffiol, R.; Plain, J.; Royer, P. Nonradiative Excitation Fluorescence: Probing Volumes Down to the Attoliter Range. J. Phys. Chem. Lett. 2010, 1, 24512454.

(20) Riachy, L.; Vézy, C.; Jaffiol, R. Non-radiative excitation fluorescence microscopy. Proc.SPIE 2016, 9721, $9721-9721-8$.

(21) Limozin, L.; Sengupta, K. Quantitative reflection interference contrast microscopy (RICM) in soft matter and cell adhesion. ChemPhysChem 2009, 10, 2752-2768.

(22) Wong, A. P.; Groves, J. T. Molecular topography imaging by intermembrane fluorescence resonance energy transfer. Proc. Natl. Acad. Sci. U. S. A. 2002, 99, 14147-14152.

(23) Weisenburger, S.; Boening, D.; Schomburg, B.; Giller, K.; Becker, S.; Griesinger, C.; Sandoghdar, V. Cryogenic optical localization provides 3D protein structure data with Angstrom resolution. Nature Methods 2017, 14, 141-144. 
(24) Hein, B.; Willig, K. I.; Hell, S. W. Stimulated emission depletion (STED) nanoscopy of a fluorescent protein-labeled organelle inside a living cell. Proceedings of the National Academy of Sciences 2008, 105, 14271-14276.

(25) Bourg, N.; Mayet, C.; Dupuis, G.; Barroca, T.; Bon, P.; Lécart, S.; Lévêque-Fort, S. Direct optical nanoscopy with axially localized detection. Nature Photonics 2015, 9, $587-593$.

(26) Deschamps, J.; Mund, M.; Ries, J. 3D superresolution microscopy by supercritical angle detection. Opt. Express 2014, 22, 29081-29091.

(27) Winterflood, C. M.; Ruckstuhl, T.; Verdes, D.; Seeger, S. Nanometer Axial Resolution by Three-Dimensional Supercritical Angle Fluorescence Microscopy. Phys. Rev. Lett. 2010, 105, 108103.

(28) Kanchanawong, P.; Shtengel, G.; Pasapera, A. M.; Ramko, E. B.; Davidson, M. W.; Hess, H. F.; Waterman, C. M. Nanoscale architecture of integrin-based cell adhesions. Nature 2010, 468, 580-584.

(29) Baronsky, T.; Ruhlandt, D.; Brückner, B. R.; Schäfer, J.; Karedla, N.; Isbaner, S.; Hähnel, D.; Gregor, I.; Enderlein, J.; Janshoff, A.; Chizhik, A. I. Cell-Substrate Dynamics of the Epithelial-to-Mesenchymal Transition. Nano Letters 2017, 17, 3320-3326.

(30) Jasieniak, J.; Smith, L.; Embden, J. V.; Mulvaney, P.; Califano, M. Re-examination of the Size-Dependent Absorption Properties of CdSe Quantum Dots. 2009, 19468-19474.

(31) Strickler, S. J.; Berg, R. A. Relationship between Absorption Intensity and Fluorescence Lifetime of Molecules. The Journal of Chemical Physics 1962, 37, 814-822.

(32) Gong, K.; Zeng, Y.; Kelley, D. F. Extinction coefficients, oscillator strengths, and radiative lifetimes of $\mathrm{CdSe}, \mathrm{CdTe}$, and $\mathrm{CdTe} / \mathrm{CdSe}$ nanocrystals. J. Phys. Chem. C 2013, 117, 20268-20279. 
(33) Gong, K.; Martin, J. E.; Shea-Rohwer, L. E.; Lu, P.; Kelley, D. F. Radiative lifetimes of zincblende CdSe/CdS quantum dots. J. Phys. Chem. C 2015, 119, 2231-2238.

(34) Aubret, A.; Pillonnet, A.; Houel, J.; Dujardin, C.; Kulzer, F. CdSe/ZnS quantum dots as sensors for the local refractive index. Nanoscale 2016, 8, 2317-2325.

(35) Lukosz, W.; Kunz, R. E. Light emission by magnetic and electric dipoles close to a plane interface I Total radiated power. J. Opt. Soc. Am. 1977, 67, 1607.

(36) Chizhik, A. I.; Gregor, I.; Enderlein, J. Quantum Yield Measurement in a Multicolor Chromophore Solution Using a Nanocavity. Nano Letters 2013, 13, 1348-1351.

(37) Collot, M.; Kreder, R.; Tatarets, A. L.; Patsenker, L. D.; Mely, Y.; Klymchenko, A. S. Bright fluorogenic squaraines with tuned cell entry for selective imaging of plasma membrane vs. endoplasmic reticulum. Chem. Commun. 2015, 51, 17136-17139.

(38) Dale, R.; Eisinger, J.; Blumberg, W. The orientational freedom of molecular probes. The orientation factor in intramolecular energy transfer. Biophysical Journal 1979, 26, $161-193$.

(39) Axelrod, D. Carbocyanine dye orientation in red cell membrane studied by microscopic fluorescence polarization. Biophysical Journal 1979, 26, 557 - 573.

(40) Chittchang, M.; Salamat-Miller, N.; Alur, H. H.; Velde, D. G. V.; Mitra, A. K.; Johnston, T. P. Poly(L-lysine) as a model drug macromolecule with which to investigate secondary structure and microporous membrane transport, part 2: diffusion studies. Journal of Pharmacy and Pharmacology 2002, 54, 1497-1505.

(41) Chizhik, A. I.; Rother, J.; Gregor, I.; Janshoff, A.; Enderlein, J. Metal-induced energy transfer for live cell nanoscopy. Nature Photonics 2014, 8, $124-127$. 
(42) Blachon, F.; Harb, F.; Munteanu, B.; Piednoir, A.; Fulcrand, R.; Charitat, T.; Fragneto, G.; Pierre-Louis, O.; Tinland, B.; Rieu, J.-P. Nanoroughness Strongly Impacts Lipid Mobility in Supported Membranes. Langmuir 2017, 33, 2444-2453. 